International Journal of Pure and Applied Mathematics

Volume 117 No. $1 \quad 2017,173-183$

ISSN: 1311-8080 (printed version); ISSN: 1314-3395 (on-line version)

url: http://www.ijpam.eu

doi: 10.12732/ijpam.v117i1.14

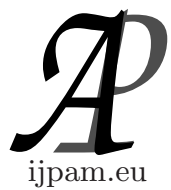

\title{
SOME NEW/OLD DEGREE-BASED TOPOLOGICAL INDICES OF NANOSTAR DENDRIMERS
}

\author{
Zeinab Foruzanfar ${ }^{1}$, Muhammad Kamran Jamil², \\ Mohammad Reza Farahani ${ }^{3} \S$, Muhammad Imran $^{4}$, Xiujun Zhang ${ }^{5}$ \\ ${ }^{1}$ Department of Engineering Sciences and Physics \\ Buein Zahra Technical University \\ Buein Zahra, Qazvin, IRAN \\ ${ }^{2}$ Department of Mathematics \\ Riphah Institute of Computing and Applied Sciences (RICAS) \\ Riphah International University \\ 14 Ali Road, Lahore, PAKISTAN \\ ${ }^{3}$ Department of Applied Mathematics \\ Iran University of Science and Technology (IUST) \\ Narmak, Tehran 16844, IRAN \\ ${ }^{4}$ Department of Mathematical Sciences \\ United Arab Emirates University \\ P.O. Box 15551, Al Ain, UNITED ARAB EMIRATES \\ ${ }^{4}$ Department of Mathematics \\ School of Natural Sciences (SNS) \\ National University of Sciences and Technology (NUST) \\ Sector H-12, Islamabad, PAKISTAN \\ ${ }^{5}$ School of Information Science and Technology \\ Chengdu University \\ Chengdu, 610106, P.R. CHINA
}

Abstract: Gutman, Furtula and Elphick reintroduced some degree based topological indices,
some of these topological indices failed to get the attention of mathematical chemist in past.

Received: 2017-09-08

Revised: 2017-10-27

Published: January 22, 2017

${ }^{\S}$ Correspondence author (c) 2017 Academic Publications, Ltd. url: www.acadpubl.eu 
These are the reciprocal Randic index (RR), the reduced reciprocal Randic index (RRR), the reduced second Zagreb index $\left(R M_{2}\right)$ and the forgotten index $(\mathrm{F})$. In this paper, we computed these indices for Nanostar Dendrimers.

AMS Subject Classification: 05C90, 05C35, 05C12

Key Words: the reduced second Zagreb index, the forgotten index, reciprocal Randic̀ index, the reduced reciprocal Randic index, Nanostar dendrimers

\section{Introduction}

Graph theory applied in the study of molecular structure represents an interdisciplinary science, called chemical graph theory or molecular topology. By using tools taken from graph theory, set theory and statistics it attempt to identify structural features involved in structure-property activity relationship. Topological indices is a subsection of chemical graph theory, which correlates certain physico-chemical properties of the underlying chemical compound. Hundreds of papers have been published on topological indices so far.

Let $\sum$ is the set of all finite simple graphs. A topological index is a function Top : $\sum \rightarrow R$ with the property that $\operatorname{Top}\left(G_{1}\right)=\operatorname{Top}\left(G_{2}\right)$, if $G_{1}$ and $G_{2}$ are isomorphic. Due to their chemical significance a lot of research has been done on topological indices of different graph families.

Nano-biotechnology is a rapidly advancing area of scientific and technological opportunity that applies the tools and processes of nano-fabrication to build devices for studying bio-systems. One of the main object of this area is Dendrimers. Dendrimers are highly ordered branched macromolecules which have attracted much theoretical and experimental attention.

In this paper, $G$ be a simple connected graph with vertex set $V(G)$ and edge set $E(G)$. The number of elements in $V(G)$ and $E(G)$ is represented as $|V(G)|$ and $|E(G)|$, respectively. For a vertex $u \in V(G)$, the number of vertices adjacent to the vertex $u$ is called the degree of $u$, denoted as $d(u)$. In 1975 Milan Randic introduced the very first vertex-degree based topological index [35], defined as

$$
R(G)=\sum_{u v \in E(G)} \frac{1}{\sqrt{d(u) d(v)}}
$$

In 1998, Bollobàs et. al. introduced the general Randic̀ index by replacing $-\frac{1}{2}$ by any real number $\alpha$ as follows,

$$
R_{\alpha}(G)=\sum_{u v \in E(G)}(d(u) d(v))^{\alpha}
$$


The first and second Zagreb indices are among the oldest and most famous topological indices, introduced by Gutman and Trinajstic [34] defined as follows

$$
\begin{aligned}
& M_{1}(G)=\sum_{v \in V(G)} d(v)^{2}=\sum_{u v \in E(G)}(d(u)+d(v)) \\
& M_{2}(G)=\sum_{u v \in E(G)} d(u) d(v)
\end{aligned}
$$

Recently, Todeschini et. al. [37, 38] proposed the multiplicative variants of ordinary Zagreb indices, defined as

$$
\begin{aligned}
& \prod_{1}(G)=\prod_{v \in V(G)} d(v)^{2} \\
& \prod_{2}(G)=\prod_{u v \in V(G)} d(u) d(v)
\end{aligned}
$$

Zhou et. al. [40] replaced the term $d(u) d(v)$ by $d(u)+d(v)$ and introduced the sum connectivity index as

$$
\chi(G)=\sum_{u v \in E(G)} \frac{1}{\sqrt{d(u)+d(v)}}
$$

and the general sum connectivity index is defined as

$$
\chi_{\alpha}(G)=\sum_{u v \in E(G)}(d(u)+d(v))^{\alpha}
$$

where $\alpha$ is any real number. For recent progress on these vertex-degree based topological indices see $[8,9,10,21,23,30,33,36,39]$

I. Gutman et. al. [22, 24] presented the neglected topological indices that earlier have been considered in the chemical and/or mathematical literature, but, that evaded the attention of most mathematical chemists. Recently, they succeed to demonstrate that these indices also have very promising applicative potential. The new/old topological indices studied by I. Gutman et. al. are the following: The reciprocal Randic̀ index is defined as

$$
R R(G)=\sum_{u v \in E(G)} \sqrt{d(u) d(v)}
$$

It is, of course, a special case of general Randic̀ index $\sum_{u v \in E(G)}(d(u) d(v))^{\alpha}$, where $\alpha$ is any real number. The reduced reciprocal Randic index is defined as

$$
R R R(G)=\sum_{u v \in E(G)} \sqrt{(d(u)-1)(d(v)-1)}
$$


The reduced second Zagreb index is defined as

$$
R M_{2}(G)=\sum_{u v \in E(G)}(d(u)-1)(d(v)-1)
$$

In a study on the structure-dependency of the total $\pi$-electron energy, beside the first Zagreb index, it was indicated that another term on which this energy depends is of the form

$$
F(G)=\sum_{v \in V(G)} d(v)^{3}=\sum_{u v \in E(G)}\left(d(u)^{2}+d(v)^{2}\right)
$$

Recently, this sum was named forgotten index, or shortly the $F$-index.

\section{Nanostar Dendrimers}

Dendrimer is a synthetic 3-dimensional macromolecule that is prepared in a stepwise fashion from simple branched monomer units. The nanostar dendrimer is a part of a new group of macromolecules that appear to photon funnels just like artificial antennas. From polymer chemistry point of view, dendrimers are nearly perfect mono-disperse macromolecules with a regular and highly branched three dimensional architecture. Some topological indices of polyomino chains are discussed in [1]-[7], [29, 32], [11]-[20]. In this section, we will compute the reciprocal Randic index, reduced reciprocal Randiindex, reduced second Zagreb index and forgotten index of nanostar dendrimers.

\subsection{Nanostar Dendrimer $N S_{1}[n], n>1$}

$N S_{1}[n]$ is the first class of these macromolecules, where $n>1$ is the defining parameter, Fig 1 . There are $140 \cdot 2^{n}-127$ edges. The technique to find the certain topological indices we partitions the edge set of graph $N S_{1}[n]$ based on degrees of end vertices of each edge.

Theorem 1. Consider $G$ be the graph of $N S_{1}[n]$, then

$$
\begin{aligned}
R R(G) & =(21+12 \sqrt{6}) 2^{n+1}-3(4 \sqrt{6}+3) \\
R R R(G) & =3(1+\sqrt{2}) 2^{n+3}-6(2 \sqrt{2}+1) \\
R M_{2}(G) & =21 \cdot 2^{n+2}-36 \\
F(G) & =129 \cdot 2^{n+2}-208
\end{aligned}
$$




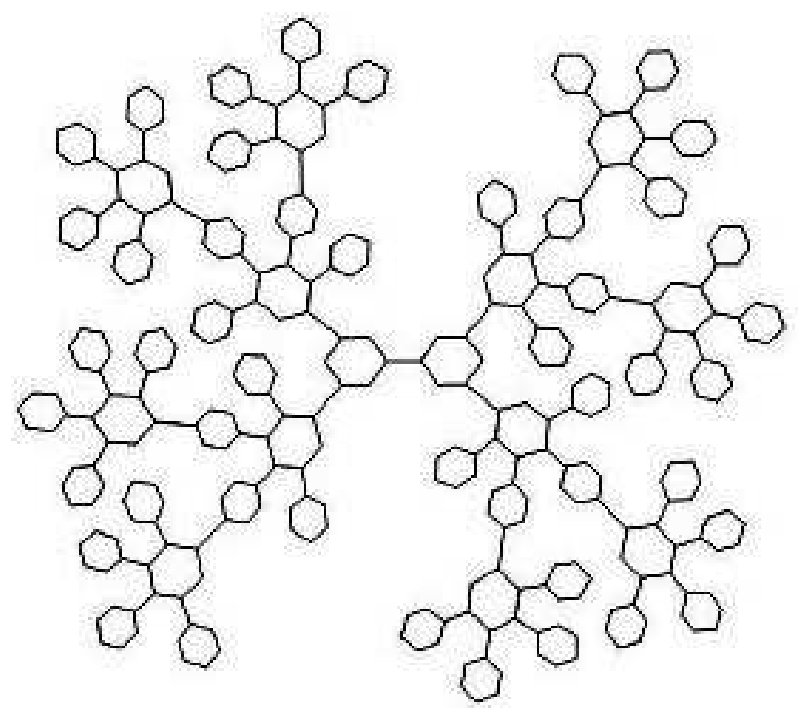

Figure 1: $N S_{1}[n]$ with $n=2$.

Proof. Consider the graph of $N S_{1}[n]$. There are three type of edges in graph of this nanostar dendrimer based on degrees of end vertices of each edge, which are

$$
\begin{aligned}
& E_{1}=\{u v \in E(G) \mid d(u)=2 \text { and } d(v)=2\} \\
& E_{2}=\{u v \in E(G) \mid d(u)=2 \text { and } d(v)=3\} \\
& E_{3}=\{u v \in E(G) \mid d(u)=3 \text { and } d(v)=3\}
\end{aligned}
$$

The number of edges in $E_{1}, E_{2}$ and $E_{3}$ are $12 \cdot 2^{n}, 24 \cdot 2^{n}-12$ and $6 \cdot 2^{n}-3$. By using these we compute $R R, R R R, R M_{2}$ and $F$ indices of $N S_{1}[n]$. Since,

$$
\begin{aligned}
R R(G) & =\sum_{u v \in E(G)} \sqrt{d(u) d(v)} \\
& =\sum_{u v \in E_{1}(G)} \sqrt{d(u) d(v)}+\sum_{u v \in E_{2}(G)} \sqrt{d(u) d(v)}+\sum_{u v \in E_{3}(G)} \sqrt{d(u) d(v)} \\
& =12 \cdot 2^{n} \sqrt{2 \cdot 2}+\left(24 \cdot 2^{n}-12\right) \sqrt{2 \cdot 3}+\left(6 \cdot 2^{n}-3\right) \sqrt{3 \cdot 3} \\
& =(21+12 \sqrt{6}) 2^{n+1}-3(4 \sqrt{6}+3)
\end{aligned}
$$

which is the required (1) result.

$$
R R R(G)=\sum_{u v \in E(G)} \sqrt{(d(u)-1)(d(v)-1)}
$$




$$
\begin{aligned}
& =\sum_{u v \in E_{1}(G)} \sqrt{(d(u)-1)(d(v)-1)}+\sum_{u v \in E_{2}(G)} \sqrt{(d(u)-1)(d(v)-1)} \\
& +\sum_{u v \in E_{3}(G)} \sqrt{(d(u)-1)(d(v)-1)} \\
& =12 \cdot 2^{n} \sqrt{(2-1)(2-1)}+\left(24 \cdot 2^{n}-12\right) \sqrt{(2-1)(3-1)} \\
& +\left(6 \cdot 2^{n}-3\right) \sqrt{(3-1)(3-1)} \\
& =3(1+\sqrt{2}) 2^{n+3}-6(2 \sqrt{2}+1)
\end{aligned}
$$

which is the required (2) result.

$$
\begin{aligned}
R M_{2}(G) & =\sum_{u v \in E(G)}(d(u)-1)(d(v)-1) \\
& =\sum_{u v \in E_{1}(G)}(d(u)-1)(d(v)-1)+\sum_{u v \in E_{2}(G)}(d(u)-1)(d(v)-1) \\
& +\sum_{u v \in E_{3}(G)}(d(u)-1)(d(v)-1) \\
& =12 \cdot 2^{n}(2-1)(2-1)+\left(24 \cdot 2^{n}-12\right)(2-1)(3-1) \\
& +\left(6 \cdot 2^{n}-3\right)(3-1)(3-1) \\
& =21 \cdot 2^{n+2}-36
\end{aligned}
$$

which is the required (3) result.

$$
\begin{aligned}
F(G) & =\sum_{u v \in E(G)}\left(d(u)^{2}+d(v)^{2}\right) \\
& =\sum_{u v \in E_{1}(G)}\left(d(u)^{2}+d(v)^{2}\right)+\sum_{u v \in E_{2}(G)}\left(d(u)^{2}+d(v)^{2}\right) \\
& +\sum_{u v \in E_{3}(G)}\left(d(u)^{2}+d(v)^{2}\right) \\
& =12 \cdot 2^{n}\left(2^{2}+2^{2}\right)+\left(24 \cdot 2^{n}-12\right)\left(2^{2}+3^{2}\right)+\left(6 \cdot 2^{n}-3\right)\left(3^{2}+3^{2}\right) \\
& =129 \cdot 2^{n+2}-208
\end{aligned}
$$

which is the required (4) result, and the proof is complete.

\subsection{Nanostar Dendrimer $N S_{2}[n], n>1$}

$N S_{2}[n]$ is the second class of these macromolecules, where $n>1$ is the defining parameter, Fig 2 . There are $140 \cdot 2^{n}-127$ edges. The technique to find the 
certain topological indices we partitions the edge set of graph $N S_{2}[n]$ based on degrees of end vertices of each edge.

Theorem 2. Consider $G$ be the graph of $N S_{2}[n]$, then

$$
\begin{aligned}
R R(G) & =(55+12 \sqrt{6}) 2^{n+2}-(201+44 \sqrt{6}) \\
R R R(G) & =(8+3 \sqrt{2}) 2^{n+4}-2(22 \sqrt{2}+59) \\
R M_{2}(G) & =37 \cdot 2^{n+3}-276 \\
F(G) & =215 \cdot 2^{n+3}-1586
\end{aligned}
$$

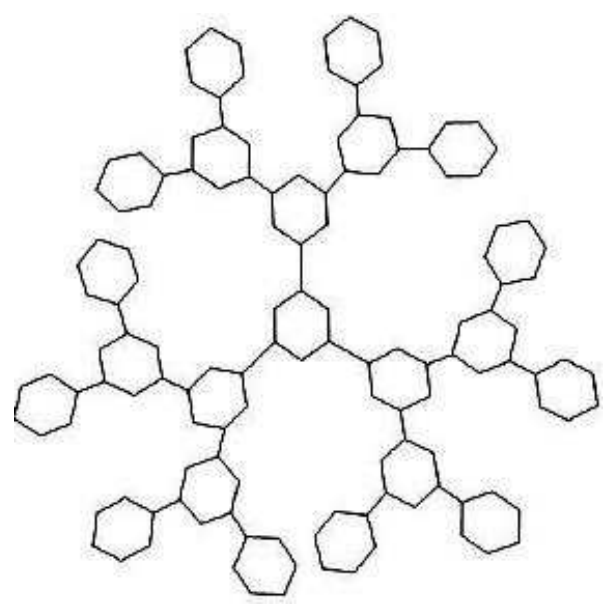

Figure 2: $N S_{2}[n]$ with $n=2$.

Proof. Consider the graph of $N S_{2}[n]$. There are three types of edges in this nanostar dendrimer's graph based on degrees of end vertices of each edge, which are

$$
\begin{aligned}
& E_{1}=\{u v \in E(G) \mid d(u)=2 \text { and } d(v)=2\} \\
& E_{2}=\{u v \in E(G) \mid d(u)=2 \text { and } d(v)=3\} \\
& E_{3}=\{u v \in E(G) \mid d(u)=3 \text { and } d(v)=3\}
\end{aligned}
$$

The number of edges in $E_{1}, E_{2}$ and $E_{3}$ are $56 \cdot 2^{n}-48,48 \cdot 2^{n}-44$ and $36 \cdot 2^{n}-35$. By using these we compute $R R, R R R, R M_{2}$ and $F$ indices of $N S_{2}[n]$. Since,

$$
R R(G)=\sum_{u v \in E(G)} \sqrt{d(u) d(v)}
$$




$$
\begin{aligned}
& =\sum_{u v \in E_{1}(G)} \sqrt{d(u) d(v)}+\sum_{u v \in E_{2}(G)} \sqrt{d(u) d(v)}+\sum_{u v \in E_{3}(G)} \sqrt{d(u) d(v)} \\
& =\left(56 \cdot 2^{n}-48\right) \sqrt{2 \cdot 2}+\left(48 \cdot 2^{n}-44\right) \sqrt{2 \cdot 3}+\left(36 \cdot 2^{n}-35\right) \sqrt{3 \cdot 3} \\
& =(55+12 \sqrt{6}) 2^{n+2}-(201+44 \sqrt{6})
\end{aligned}
$$

which is the required (5) result.

$$
\begin{aligned}
R R R(G) & =\sum_{u v \in E(G)} \sqrt{(d(u)-1)(d(v)-1)} \\
& =\sum_{u v \in E_{1}(G)} \sqrt{(d(u)-1)(d(v)-1)}+\sum_{u v \in E_{2}(G)} \sqrt{(d(u)-1)(d(v)-1)} \\
& +\sum_{u v \in E_{3}(G)} \sqrt{(d(u)-1)(d(v)-1)} \\
& =\left(56 \cdot 2^{n}-48\right) \sqrt{(2-1)(2-1)}+\left(48 \cdot 2^{n}-44\right) \sqrt{(2-1)(3-1)}+ \\
& \left(36 \cdot 2^{n}-35\right) \sqrt{(3-1)(3-1)} \\
& =(8+3 \sqrt{2}) 2^{n+4}-2(22 \sqrt{2}+59)
\end{aligned}
$$

which is the required (6) result.

$$
\begin{aligned}
R M_{2}(G) & =\sum_{u v \in E(G)}(d(u)-1)(d(v)-1) \\
& =\sum_{u v \in E_{1}(G)}(d(u)-1)(d(v)-1)+\sum_{u v \in E_{2}(G)}(d(u)-1)(d(v)-1) \\
& +\sum_{u v \in E_{3}(G)}(d(u)-1)(d(v)-1) \\
& =\left(56 \cdot 2^{n}-48\right)(2-1)(2-1)+\left(48 \cdot 2^{n}-44\right)(2-1)(3-1)+ \\
& \left(36 \cdot 2^{n}-35\right)(3-1)(3-1) \\
& =37 \cdot 2^{n+3}-276
\end{aligned}
$$

which is the required (7) result.

$$
\begin{aligned}
F(G) & =\sum_{u v \in E(G)}\left(d(u)^{2}+d(v)^{2}\right) \\
& =\sum_{u v \in E_{1}(G)}\left(d(u)^{2}+d(v)^{2}\right)+\sum_{u v \in E_{2}(G)}\left(d(u)^{2}+d(v)^{2}\right) \\
& +\sum_{u v \in E_{3}(G)}\left(d(u)^{2}+d(v)^{2}\right)
\end{aligned}
$$




$$
\begin{aligned}
& =\left(56 \cdot 2^{n}-48\right)\left(2^{2}+2^{2}\right)+\left(48 \cdot 2^{n}-44\right)\left(2^{2}+3^{2}\right)+\left(36 \cdot 2^{n}-35\right)\left(3^{2}+3^{2}\right) \\
& =215 \cdot 2^{n+3}-1586
\end{aligned}
$$

which is the required (8) result, and the proof is complete.

\section{Concluding Remarks and Open Problems}

Recently, Gutman, Furtula and Elphick introduced reciprocal Rindic̀ index, reduced reciprocal Randic̀ index, reduced second Zagreb index and forgotten index with great significant applications in chemical graph theory. In this article, we computed these topological indices for Nanostar Dendrimers. Some future work that can be done is to find these topological indices for Polyomino chains, benzenoid systems or some other chemical structures.

\section{Acknowledgments}

The authors are very grateful to the referees for their constructive suggestions and useful comments, which improved this work very much. This research was supported by Key Project of Sichuan Provincial Department of Education under grant 17ZA0079.

\section{References}

[1] A.R. Ashrafi, M. Mirzargar, PI, Szeged, and edge Szeged indices of an infinite family of Nanostar Dendrimers. Indian J. Chem, 47A (2008), 538-541.

[2] A.R. Ashrafi and P. Nikzad, Connectivity index of the family of Dendrimer Nanostars, Digest. J. Nanomater. Bios. 4(2), 269-273 (2009).

[3] A.R. Ashrafi and P. Nikzad, Kekule index and bounds of energy for Nanostar Dendrimers, Digest. J. Nanomater. Bios. 4(2), 383-388 (2009).

[4] S. Alikhani, M.A. Iranmanesh, Chromatic polynomials of some Dendrimers, J. MATCH Commun. Math. Comput. Chem. 62 (2009), 363-370.

[5] S. Alikhani, M.A. Iranmanesh, Chromatic polynomials of some Dendrimers. J. Comput. Theor. Nanosci. 7(11) (2010), 2314-2316, doi: 10.1166/jctn.2010.1613.

[6] S. Alikhani, M.A. Iranmanesh, Chromatic polynomials of some Nanostars, Iranian Journal of Math. Chemist. 3(2) (2010), 127-135.

[7] S. Alikhani, M.A. Iranmanesh, Eccentric connectivity polynomials of an infinite family of Dendrimers. Digest. J. Nanomater. Bios. 6(1) (2011), 256-257. 
[8] K. C. Das, I. Gutman, Some properties of the second Zagreb index, MATCH Commun. Math. Comput. Chem. 52 (2004) 103-112.

[9] K. C. Das, I. Gutman, B. Furtula, Survey on geometric-arithmetic indices of graphs, MATCH Commun. Math. Comput. Chem. 65 (2011) 595-644.

[10] T. Doslic̀, B. Furtula, A. Graovac, I. Gutman, S. Moradi, Z. Yarahmadi, On vertexdegree-based molecular structure descriptors, MATCH Commun. Math. Comput Chem. 66 (2011) 613-626.

[11] M.R. Farahani, Fourth Atom-bond connectivity index of an infinite class of Nanostar Dendrimer $D_{3}[n]$, Journal of Advances in Chemistry, 4(1) (2013), 301-305.

[12] M.R. Farahani, Computing Fifth Geometric-Arithmetic Index of Dendrimer Nanostars, Advances in Materials and Corrosion. 1 (2013), 62-64.

[13] M.R. Farahani, Some connectivity index of an infinite class of Dendrimer Nanostars. Journal of Applied Physical Science International, 3(3), 2015, 99-105.

[14] M.R. Farahani. On Multiple Zagreb indices of Dendrimer Nanostars. International Letters of Chemistry, Physics and Astronomy. 52 (2015), 147-151.

[15] M.R. Farahani, W. Gao, M.R. Rajesh Kanna. The Connective Eccentric Index for an Infinite Family of Dendrimers. Indian Journal of Fundamental and Applied Life Sciences. 2015, 5(S4), 766-771.

[16] M.R. Farahani, A.Q. Baig, W. Sajjad. Second Eccentric Zagreb Index on Infinite family of Nanostar Dendrimers. Journal of Mathematical Nanoscience. 2017, In press.

[17] W. Gao, M.R. Farahani. The Hyper-Zagreb Index for an infinite family of Nanostar Dendrimer. Journal of Discrete Mathematical Sciences and Cryptography. 2017, In press, doi: 10.1080/09720529.2016.1220088.

[18] W. Gao, M.R. Farahani, M.R. Rajesh Kanna. The Multiplicative Zagreb Indices of Nanostructures and Chains. Open Journal of Discrete Mathematics. 6, 2016, 82-88, doi: 10.4236/ojdm.2016.62008.

[19] D.X. Li, J.B. Liu, M. Rezaei, M.R. Farahani. Zagreb Indices and Zagreb Polynomials of an Infinite Class of Dendrimer Nanostars. Journal of Computational and Theoretical Nanoscience. 13(12), 2016, 9136-9139, doi: 10.1166/jctn.2016.6293.

[20] L. Yan, Y. Li, M.R. Farahani, A.N. Al-Kenani, M.R. Rajesh Kanna, R. Pradeep Kumar. The Ediz Eccentric Connectivity index of Nanostar Dendrimer $D_{3}[n]$. International Journal of Biology, Pharmacy and Allied Sciences. 5(7), 2016,1591-1596.

[21] I. Gutman, K. C. Das, The first Zagreb index 30 years after, MATCH Commun. Math. Comput. Chem. 50 (2004) 83-92.

[22] I. Gutman, D. Dimitrov, H. Abdo, On extremal trees with respect to the F-index, arXive: 1509303574 v1.

[23] I. Gutman, B. Furtula (Eds.), Novel Molecular Structure Descriptors - Theory and Applications I, Univ. Kragujevac, Kragujevac, 2010.

[24] I. Gutman, B. Furtula, C. Elphick, Three new/old vertex-degree based topological indices, MATCH Commun. Math. Comput. Chem., 72 (2014) 617-632.

[25] M. Ghorbani, M. Ghazi, Computing some topological indices of triangular benzenoid, Digest Journal of Nanomaerials and Biostructures, 5,4 (2010) 1107-1111. 
[26] I. Gutman, M. K. Jamil, N. Akhter, Graphs with fixd number of pendent vertices and minimal first Zagreb index, Tanssaction on Combinatorics, 41 (2015) 43-48.

[27] N.M. Husin, R. Hasni, N.E. Arif, Atom-Bond Connectivity and Geometric Arithmetic Indices of Dendrimer Nanostars, Australian Journal of Basic and Applied Sciences, 7(9) (2013), 10-14.

[28] Y. Huo, J. B. Liu, A.Q. Baig, W. Sajjad, M.R. Farahani, Connective Eccentric Index of $N A_{m}^{n}$ Nanotube, Journal of Computational and Theoretical Nanoscience, 14(4), 18321836, 2017, doi: $10.1166 /$ jctn.2017.6512.

[29] M. Imran, S. Hayat, M. K. Shafiq, On topological indices of nanostar dendrimers and poloyomino chains, Optoelectronics and Advanced Materials-Rapid Communications, 8 (2014) 948-954.

[30] M. K. Jamil, I. Tomescu, N. Akhter, Extremal degree-product indices of graphs with fixed number of pendant vertices and cyclomatic number, International Letters of Chemistry, Physics and Astronomy, 59 (2015) 53-61, doi: 10.18052/www.scipress.com/ILCPA.59.53.

[31] D. A. Klarner, Polyominoes, In: J. E. Goodman, J. O'Rourke (eds.) Handbook of Discrete and Computational Geometry, CRC Press, Boca Raton Chapter 12 (1997) 225-242.

[32] M. Mirzargar, PI, Szeged and edge Szeged polynomails of dendrimer nanostar, MATCH Commun. Math. Comput. Chem. 62 (2009) 363-370.

[33] S. Nikolic̀, G. Kovačevic̀, A. Miličevic̀, N. Trinajstic̀, The Zagreb indices 30 years after, Croat. Chem. Acta 76 (2003) 113-124.

[34] I. Gutman, N. Trinajsti; Graph theory and molecular orbitals, Total $\pi$-electron energy of alternant hydrocarbons, Chemical Physics Letters. 17 (1972) 535-538, doi: 10.1016/00092614(72)85099-1.

[35] M. Randic̀, On characterization of molecular branching, J. Am. Chem. Soc. 97 (1975) 6609-6615, doi: 10.1021/ja00856a001.

[36] M. Randic̀, The connectivity index 25 years after, Journal of Molecular Graphics and Modelling. 20 (2001) 19-35.

[37] R. Todeschini, D. Ballabio, V. Consonni, Novel molecular descriptors based on functions of new vertex degrees. In: Gutman, I., Furtula, B. (eds.), Novel Molecular Structure Descriptors-Theory and Applications, Univ. Kragujevac, Kragujevac, 1 (2010) 73-100.

[38] R. Todeschini, V. Consonni, New local vertex invariants and molecular descriptors based on functions of the vertex degrees, MATCH Commun. Math. Comput. Chem., 64 (2010) 359-372.

[39] I. Tomescu, M. K. Jamil, Maximum general sum-connectivity index for trees with given independence number, MATCH Commun. Math. Comput. Chem. 72 (2014) 715-22.

[40] B. Zhou, N. Trinajstic̀, On a novel connectivity index, J. Math. Chem., 46 (2009) 12521270, doi: 10.1007/s10910-008-9515-z. 
